\title{
REGULATION OF OCULAR IMMUNE RESPONSES
}

\author{
J. WAYNE STREILEIN \\ Boston, Massachusetts
}

\section{SUMMARY}

The existence of immune privilege in numerous tissues and organs, including the eye, has been re-emphasised during the past few years, and experimental studies have begun to unravel the multiple mechanisms, both passive and active, that make privilege possible. In this review, recent evidence bearing on the factors responsible for immune privilege in the anterior chamber of the eye is described. One dimension of ocular immune privilege depends upon local factors that limit and modify the expression of immunity. As a consequence, the local expression of immunogenic inflammation is largely curtailed within the eye. Another dimension of ocular immune privilege concerns the modification of induction of systemic immunity to antigens placed within, or arising from, the eye. Systemic responses to ocular antigens are devoid of delayed hypersensitivity $T$ cells and complement-fixing antibodies, and the stage for these stereotypic responses is set by factors within the ocular microenvironment acting on indigenous antigen presenting cells. Overall, regulation of immune responses directed at ocular antigens appears to be designed to prevent inflammation from disrupting the visual axis and thereby causing blindness.

No organism and no tissue can survive without the aid of the immune system to recognise and eliminate pathogens. Yet the immune system possesses the potential not only to heal but to cause disease. Immunopathology is the study of mechanisms by which immune responses lead to tissue damage and dysfunction. The reason immune responses do not necessarily, or even usually, produce pathology is because the response itself is highly regulated, and regulation takes place in every organ or tissue in which immunity expresses itself. The eye is no exception to this rule, and the phenomenon of immune privilege is a dramatic example of tissuespecific regulation of immune responsiveness.

Correspondence to: J. Wayne Streilein, Schepens Eye Research Institute, Harvard Medical School, 20 Staniford Street, Boston, MA 02114, USA.

\section{OCULAR IMMUNE PRIVILEGE}

Immune privilege in the eye was discovered experimentally more than a century ago, and can be defined in modern terms as follows: ${ }^{1-3}$ foreign tissues placed into various intraocular compartments (corneal stroma, anterior chamber, vitreous cavity, subretinal space) experience prolonged, often indefinite, survival compared with foreign tissues placed at conventional sites. Since immune rejection is the mechanism by which foreign tissue grafts are typically destroyed, then regulation of the rejection mechanism by the eye is responsible for unexpected graft survival within ocular compartments.

It is worth considering why immune privilege exists in the eye, or any other so-called privileged tissue. Our view is that all tissues, including those with special physiological needs, require immune protection. Immune privilege represents the consequence of interactions between the immune system and specialised tissues in which local protection is provided by immune effectors that do not disrupt differentiated tissue function. In the case of the eye, a precise microanatomy must be maintained in order for vision to be preserved. Delicate ocular structures cannot tolerate intense inflammation without losing integrity and vision. Immune privilege allows for ocular immunity that is protective but virtually devoid of immunogenic inflammation.

Immune-privileged tissues and sites possess a range of features that are important in the creation and maintenance of the privileged state. In general, these features can be considered as 'passive' and 'active', as listed in Table I. Passive features represent stable components and aspects of the eye. The blood-ocular barrier, residing at the edge of the cornea, among iris vessels, the epithelium of the ciliary body, retinal pigment epithelium and the retinal vessels, regulates stringently the access of blood-borne cells and molecules into the eye. Immune effectors within the blood stream, including sensitised $\mathrm{T}$ cells and antibodies, are largely (but not completely) excluded from the eye by this potent barrier. Expression of immunity in the eye is thus 
Table I. Features of immune-privileged sites

Passive

Blood-tissue barriers

Deficient efferent lymphatic drainage

Tissue fluid that drains into blood vasculature

Reduced expression of MHC class I and II on parenchymal cells

Active

Constitutive expression of cell surface molecules that modulate immune effector cells and molecules: atypical class Ib

Fas ligand

CD59, MCP, DAF - inhibitors of complement

Immunosuppressive microenvironment containing:

transforming growth factor-beta

alpha-melanocyte stimulating hormone

vasoactive intestinal peptide

calcitonin gene-related peptide

free cortisol

IL-1 receptor antagonist

controlled passively. In addition, the eye (at least its internal compartments) lacks easily demonstrable lymphatic drainage pathways. Since lymph vessels represent the primary pathway by which most antigenic substances first encounter $\mathrm{T}$ and $\mathrm{B}$ cells in organised lymphoid tissues, the lack of lymphatics prevents ocular antigens from sensitising in a conventional fashion. Instead, antigenic information escapes from the eye largely into the venous circulation, because intraocular fluids are drained via the trabecular meshwork into the canals of Schlemm. Besides these microanatomical features, parenchymal cells of the eye express poorly, or not at all, class I and class II molecules encoded within the major histocompatibility complex (MHC). ${ }^{4}$ Virtually no class II molecules exist in the cornea, nor are they expressed on the epithelial cells surrounding the anterior chamber, vitreous cavity or subretinal space. Class I expression is very sparse on corneal endothelial cells and cells of the neuronal retina. Since MHC molecules are required for $\mathrm{T}$ cells to detect antigenic substances, reduced expression of these molecules on ocular cells reduces the ability of $\mathrm{T}$ cells to detect antigens within ocular compartments.

Active features of immune privilege correspond to molecules that are uniquely expressed by ocular cells, molecules that profoundly influence the behaviour of cells of the immune system. Ocular parenchymal cells have been found to express constitutively Fas ligand, ${ }^{5}$ and membrane inhibitors of activated complement components ${ }^{6}$ (Table I). The latter interfere with completion of the complement cascade (and therefore prevent immune-mediated cell lysis). Fas ligand binds to its co-receptor Fas, which is expressed on activated $\mathrm{T}$ cells, and polymorphonuclear leucocytes. Among certain $\mathrm{Fas}^{+}$ cells, Fas ligation induces apoptosis. It has been proposed that this mechanism may cause antigenspecific $T$ cells to be deleted when they encounter antigen within the eye. In addition to these mem- brane-bound molecules, the soluble intraocular microenvironment (as revealed by aqueous humour) contains a variety of factors that suppress and regulate immune cell function. The list includes transforming growth factor-beta 2 (TGF $\beta-2)$ alphamelanocyte stimulating hormone, vasoactive intestinal peptide, calcitonin gene-related peptide, free cortisol and interleukin-1 receptor antagonist. ${ }^{7,8}$

It is too early to describe with precision the means by which these active and passive features conspire to produce immune privilege in the eye. It is helpful and reasonable to consider eye-derived regulatory forces as acting at one of two critical stages in development of the immune response: first, during induction of immunity, usually referred to as the afferent limb of the immune reflex arc; and second, during expression of immunity, referred to as the efferent limb. Recent experiments have begun to reveal certain aspects of ocular regulation that influence immune induction, and other aspects that modify immune expression.

\section{REGULATION OF IMMUNE EXPRESSION IN THE EYE}

In vitro studies of aqueous humour offer one approach to examining the ways in which the eye influences immune expression. Our laboratory initiated studies on the effects of aqueous humour on $\mathrm{T}$ cell activation almost 10 years ago, and those studies have continued to bear fruit under the direction of Dr Andrew Taylor. Kaiser et al. ${ }^{9}$ demonstrated in 1988 that murine and rabbit aqueous humour inhibited $\mathrm{T}$ cell activation and therefore suppressed proliferation and lymphokine production. Taylor has refined those studies by exposing in vivo primed, TB-specific $\mathrm{T}$ cells to an anti-CD3 antibody that stimulates the cells to secrete interferon-gamma (IFN- $\gamma$ ). When anti-CD3 stimulation takes place in the presence of aqueous humour, IFN- $\gamma$ secretion by these T cells comes to a halt. ${ }^{10}$ Evidence indicates that the major aqueous humour 
Table II. Influence of transforming growth factor-beta on immune induction via the anterior chamber of the eye

\begin{tabular}{ll}
\hline Cell type & Effects \\
\hline Antigen presenting cells & $\begin{array}{l}\text { Induce cells to secrete mature TGF } \beta \\
\text { Shift processing of antigen so that peptides are loaded preferentially on class I, rather than class II molecules }\end{array}$ \\
T lymphocytes & $\begin{array}{l}\text { Induce cells to secrete TGF } 3 \\
\text { Inhibit secretion of pro-inflammatory lymphokines }\end{array}$ \\
\hline
\end{tabular}

factor responsible for inhibiting IFN- $\gamma$ production is TGF $\beta$ (Table II). This effect probably accounts for the observation of Cousins et al. ${ }^{11}$ in which tuberculin-specific $\mathrm{T}$ cells capable of mediating delayed hypersensitivity in vivo were inhibited from doing so when first exposed to aqueous humour. Since IFN- $\gamma$ appears to be the key cytokine responsible for delayed hypersensitivity responses in vivo, and since aqueous humour prevents activated $\mathrm{T}$ cells from producing this cytokine, the eye can prevent intraocular expression of delayed hypersensitivity if antigen is present.

The ability of the eye to modify immune expression is not limited to cell-mediated processes. Over the past 30 years scattered reports have claimed that aqueous humour inhibits complement activation. ${ }^{12}$ Our laboratory (W. Goslings) has recently initiated studies to explore this possibility in greater depth. Using assays that enable one to examine selectively activation of complement via the classical and alternative pathways, Goslings has found that aqueous humour contains at least two distinct anticomplementary activities. Fractionation of normal rabbit aqueous humour by size filtration reveals two peaks of adsorption at $280 \mathrm{~nm}$. One peak is quite broad and covers molecules of 12000 daltons and greater; this peak contains small amounts of many proteins derived from blood plasma, and it is within this fraction that the factor(s) that inhibits the alternative pathway resides. The other peak includes molecules of less than 3000 daltons, and within this peak resides another factor that profoundly inhibits activation of complement via antibody. What is particularly interesting about this fraction is that the inhibiting component appears to have a molecular weight of 1000 or less, and is not a protein. Review of the literature indicates that aminated sugars, such as glucosamine, have the ability to suppress complement activation, and the relevant fraction in aqueous humour is known to contain such sugars. Experiments are now under way to identify the relevant molecules. In addition, constitutive expression of CD59, MCP and DAF on cells that surround the anterior chamber, described recently by Bora et al. ${ }^{6}$ (Table I), further indicates the potential capacity of this microenvironment to nullify the deleterious effects of complement activation in the eye.

These two lines of investigation demonstrate that aqueous humour possesses the capacity to inhibit the expression of immunity, especially those effectors with the potential to evoke vigorous inflammation. Delayed hypersensitivity and antibody-dependent complement fixation are both characterised by intense inflammation. Their inhibition by aqueous humour emphasises that immune privilege depends in part upon avoidance of immunogenic inflammation as a way of preserving vision.

\section{REGULATION OF IMMUNE INDUCTION VIA THE EYE}

Antigenic material introduced into the anterior chamber is not ignored by the systemic immune apparatus. Instead, intraocular antigen elicits a stereotypic, systemic immune response that is selectively deficient in $T$ cells that mediate delayed hypersensitivity, and $B$ cells that secrete complement-fixing antibodies. At the same time, other types of $\mathrm{T}$ cells are activated, including regulatory $\mathrm{T}$ cells and precursors of cytotoxic $\mathrm{T}$ cells, and $\mathrm{B}$ cells are also generated - cells that produce non-complementfixing antibodies. This deviant form of systemic immunity has been termed Anterior Chamber Associated Immune Deviation (ACAID), and current evidence indicates that ACAID is a central feature of ocular immune privilege..$^{2,13,14}$ Our laboratory has described one interesting pathway by which antigens introduced into the eye induce ACAID. ${ }^{15-17}$ Although soluble antigenic molecules injected into the anterior chamber rapidly escape into the blood, native antigen injected intravenously does not induce ACAID. Instead, the antigenic moieties that induce ACAID are first captured by indigenous ocular antigen presenting cells (APC; dendritic cells/macrophages) that normally reside in the stroma and epithelium, iris and ciliary body. Upon anterior chamber injection of antigen, intraocular APC endocytose the material, migrate across the trabecular meshwork into the venous system, and then traffic selectively to the white pulp of the spleen. Within this site the eye-derived cells come to rest. APC with identical properties have been generated in vitro by incubating conventional APC with antigen overnight in the presence of aqueous humour. When injected intravenously into naive recipients, such cells also migrate preferentially to the spleen. It has been demonstrated that the active ingredient in aqueous humour is TGF $\beta$; incubation of APC with TGF $\beta$ alone has exactly the same ACAID-conferring effect on APC. With the aid of recombinant inbred strains of mice, $\mathrm{MHC}$ restriction experiments have demonstrated that APC pulsed with antigen in 
the presence of aqueous humour act as the proximate APC in ACAID. ${ }^{18}$ These same experimental data suggest that class I rather than class II MHC molecules are more important in ACAID induction. This is of considerable interest because the spectrum of $\mathrm{T}$ cells activated in the spleens of mice with ACAID includes predominantly $\mathrm{CD} 8^{+}$cytotoxic $\mathrm{T}$ cell precursors, and $\mathrm{CD}^{+}$suppressor cells; scant evidence of activation of $\mathrm{CD}^{+} \mathrm{T}$ cells can be found. ${ }^{19}$

Within the past year, the $\mathrm{T}$ cell activating properties of in vitro generated ACAID-inducing APC have been studied in our laboratory by $\operatorname{Dr} \mathrm{M}$. Takeuchi. ${ }^{20}$ The results confirm and extend our understanding of this process (Table II). Dr Takeuchi has taken advantage of Tcr transgenic mice in whom the majority of $\mathrm{T}$ cells express a receptor for the immunodominant peptide of ovalbumin (P323-339) in the context of I- $\mathrm{A}^{\mathrm{d}}$. Culture of these $\mathrm{T}$ cells with peritoneal exudate cells (PEC) enriched for macrophages that have been pulsed with ovalbumin itself, or with P323-339, results in proliferation, and secretion of interleukin-2 (IL-2) and IFN- $\gamma$. However, when the PEC are first incubated with TGF $\beta-2$ (the isotype found in normal aqueous humour) and are then pulsed with either ovalbumin or P323-339, $\mathrm{T}$ cell activation fails. Takeuchi has found at least two reasons for this failure. On the one hand, PEC exposed to TGF $\beta-2$ begin to secrete large amounts of mature TGF $\beta$. This cytokine is a strong inhibitor of $T$ cell activation, and Takeuchi has found that neutralising anti-TGF $\beta$ antibody in the culture enables transgenic $T$ cells to respond to P323-339-pulsed, TGF $\beta$-treated PEC. However, this antibody does not restore the ability of these transgenic $T$ cells to respond when TGF $\beta$ treated PEC are pulsed with native ovalbumin. The reason for this failure may reside in the results of a separate experiment in which $\mathrm{T}$ cell hybridoma cells, with Tcr specific for an ovalbumin-derived peptide in the context of a class I molecule, are confronted by PEC pulsed with ovalbumin. Whereas a modest degree of hybridoma stimulation (IL-2 secretion) occurs when the cells are exposed to untreated PEC pulsed with ovalbumin, significantly more IL-2 is secreted by hybridoma cells confronted with ovalbumin-pulsed PEC pretreated with TGF $\beta-2$. These findings suggest that PEC treated with TGF $\beta$ degrade exogenous protein antigens into peptides that are loaded preferentially on class I rather than class II molecules for presentation to $\mathrm{T}$ cells. Although further experiments are needed to provide definitive proof, in their current form the data support the hypothesis that the eye, via TGF $\beta$, alters the antigen processing and presenting potential of professional antigen presenting cells, and induces the cells to secrete immunosuppressive TGF $\beta$. In this setting, $\mathrm{CD} 8^{+} \mathrm{T}$ cells are preferentially activated.

The functions of $T$ cells activated in the presence of aqueous humour have been evaluated recently by Dr A. W. Taylor, and some of the results are pertinent to this line of reasoning. ${ }^{21} \mathrm{Dr}$ Taylor has found that primed $\mathrm{T}$ cells exposed to Tcr ligand in the presence of aqueous humour are induced to secrete their own TGF $\beta-1$ (Table II). Production of this immunoinhibitory cytokine by $\mathrm{T}$ cells has important consequences. If $\mathrm{T}$ cells previously activated in the presence of aqueous humour are cocultured with freshly prepared primed $\mathrm{T}$ cells stimulated with Tcr ligand, the freshly prepared $\mathrm{T}$ cells fail to secrete IFN- $\gamma$. The reason for this failure is revealed by experiments in which neutralising soluble TGF $\beta$-receptor 2 is added to identical cultures. Under this circumstance, IFN- $\gamma$ secretion is restored. While much remains to be explored in this intriguing system, the data at this point suggest that aqueous humour not only induces primed $\mathrm{T}$ cells to secrete TGF $\beta$, but that secretion of this cytokine in this microenvironment suppresses the activation of bystander fresh $\mathrm{T}$ cells exposed to Tcr ligand. We are considering the possibility that at least one population of suppressor cells generated in ACAID has the phenotype of secretion of TGF $\beta$ upon exposure to antigen.

Thus, the ocular microenvironment modifies the induction of immunity to eye-derived antigens. Aqueous humour confers functional properties upon resident APC (secretion of TGF $\beta$, loading of peptides of antigen on class I molecules) which ensure that responding, antigen-specific $\mathrm{T}$ cells are $\mathrm{CD}^{+}$(class I restricted) and produce TGF $\beta$ rather than pro-inflammatory cytokines.

\section{SUMMARY AND CONCLUSIONS}

Immune responses to antigens within the eye are highly regulated. Immune privilege is a dramatic expression of that regulation. Privilege is a dynamic, actively acquired state, rather than a passive one. Active processes regulate both the induction of immunity to antigens introduced into, or arising from, the eye, and the expression of immunity in the eye. The rationale of these regulatory mechanisms appears to be related to the need for the eye to avoid the deleterious consequences of immunopathogenic injury, especially that due to immunogenic inflammation. Among the active mechanisms employed by the eye to secure immune privilege is a microenvironment enriched for immunomodulatory cytokines (such as TGFB-2) and inhibitors of complement activation, and for constitutive expression of cell surface molecules (Fas ligand, CD59, MCP, DAF) that can abort the activation of effector $T$ cells and the ability of antibodies to fix complement. Not only 
do these ocular mechanisms inhibit the expression of immunogenic inflammation within the eye, but they act via ACAID to alter the very essence of systemic immune responses to eye-derived antigens. Through the actions of TGF $\beta$ on APC directly, and on T cells indirectly, systemic immune responses to ocular antigens are mediated chiefly by class I-restricted $\mathrm{CD} 8^{+} \mathrm{T}$ cells, among which are suppressor cells that mediate their effects through production of their own TGFß. In this manner, the 'gestalt' of immune privilege is transferred from the ocular microenvironment to $T$ cells that recognise eye-derived antigens. As a consequence, metastatic immune privilege is created wherever these cells encounter the same antigens - whether in the eye or elsewhere.

Key words: Immune deviation, Immune privilege, Suppression, Inflammation, Delayed hypersensitivity, Microenvironment.

\section{REFERENCES}

1. Barker CF, Billingham RE. Immunologically privileged sites. Adv Immunol 1977;25:1-54.

2. Streilein JW. Ocular regulation of systemic immunity. Reg Immunol 1994;6:143-50.

3. Ksander BR, Streilein JW. Regulation of the immune response within privileged sites. In: Granstein $\mathrm{R}$, editor. Mechanisms of regulation of immunity. Basel: Karger, 1993:177-45.

4. Abi-Hanna D, Wakefield D, Watkins S. HLA antigens in ocular tissues. I. In vivo expression in human eyes. Transplantation 1988;45:610-3.

5. Griffith TS, Brunner T, Fletcher SM, Green DR, Ferguson TA. Fas ligand-induced apoptosis as a mechanism of immune privilege. Science 1995;270: 1189-92.

6. Bora NS, Gobleman CL, Atkinson JP, Pepose JS, Kaplan HJ. Differential expression of the complement regulatory proteins in the human eye. Invest Ophthalmol Vis Sci 1993;34:3579-84.

7. Streilein JW. Immune privilege as the result of local tissue barriers and immunosuppressive microenvironments. Curr Opin Immunol 1993;5:428-32.

8. Kennedy MC, Rosenbaum JT, Brown J, et al. Novel production of interleukin-1 receptor antagonist peptides in normal human cornea. $J$ Clin Invest 1995;95:82-9.

9. Kaiser CJ, Ksander BR, Streilein JW. Inhibition of lymphocyte proliferation by aqueous humor. Reg Immunol 1989;2:42-9.

10. Taylor AW, Streilein JW, Cousins SW. Alpha melanocyte stimulating hormone $(\alpha-\mathrm{MSH})$ suppresses antigen-stimulated $\mathrm{T}$ cell production of IFN- $\gamma$. NeuroImmunoModulation 1994;1:188-94.

11. Cousins SW, Trattler WB, Streilein JW. Immune privilege and suppression of immunogenic inflammation in the anterior chamber of the eye. Curr Eye Res 1991;10:287-97.

12. Shimada K. The complement components and their inhibitors in the intraocular fluids of the guinea pig. Invest Ophthalmol 1970;9:304-11.

13. Tompsett E, Abi-Hanna D, Wakefield D. Immunological privilege in the eye: a review. Curr Eye Res 1990;9:1141-50.

14. Niederkorn JY, Streilein JW, Shadduck JA. Deviant immune responses to allogeneic tumors injected intracamerally and subcutaneously in mice. Invest Ophthalmol Vis Sci 1981;20:355-63.

15. Wilbanks GA, Streilein JW. Studies on the induction of Anterior Chamber-Associated Immune Deviation (ACAID). I. Evidence that an antigen-specific, ACAID-inducing, cell associated signal exists in the peripheral blood. J Immunol 1991;146:2610-7.

16. Wilbanks GA, Mammolenti MM, Streilein JW. Studies on the induction of Anterior Chamber-Associated Immune Deviation (ACAID). II. Eye-derived cells participate in generating blood borne signals that induce ACAID. J Immunol 1991;146:3018-24.

17. Wilbanks GA, Mammolenti MM, Streilein JW. Studies on the induction of Anterior Chamber-Associated Immune Deviation (ACAID). III. Induction of ACAID depends upon intraocular transforming growth factor- $\beta$. Eur J Immunol 1992;22:165-73.

18. Hara Y, Okamoto S, Rouse B, Streilein JW. Evidence that peritoneal exudate cells cultured with eye-derived fluids are the proximate antigen presenting cells in immune deviation of the ocular type. J Immunol 1993;151:162-71.

19. Bando Y, Ksander BR, Streilein JW. Characterisation of specific $\mathrm{T}$ helper cell activity in mice bearing alloantigen tumors in the anterior chamber of the eye. Eur J Immunol 1991;21:1923-32.

20. Takeuchi M, Kosiewicz MM, Streilein JW. On the mechanisms by which TGF $\beta$ alters functional properties of antigen presenting cells and $\mathrm{T}$ cells. Invest Ophthalmol Vis Sci 1996;37:S533.

21. Taylor AW, Streilein JW. Aqueous humor induces effector T cells to mediate immunosuppression. Invest Ophthalmol Vis Sci 1996;37:S1113. 